\title{
Laboratory Test of a Visual Sputum Suctioning System
}

\author{
Jingjing Lv MD, Jingxing Wu MD PhD, Rui Guo MD PhD, \\ Xun Liu MD, Bingbing Yan MD, and Huisheng Deng MD PhD
}

\begin{abstract}
BACKGROUND: Conventional sputum suctioning is a routine clinical practice, but complications may arise from the blind manipulation of the catheter. Recently, a visual sputum suctioning system (VSSS) was developed, and we tested this new system in a laboratory setting. METHODS: We used coagulant concentrations of $1.5 \%$ and $3.0 \%$ to simulate mucus and sputum. Conventional singlelumen and triple-lumen catheters were inserted separately into a beaker for sputum suctioning $(15 \mathrm{~s}, 200 \mathrm{~mm} \mathrm{Hg})$. A micro-imaging fiber was integrated into the triple-lumen catheter to create the VSSS. The single-lumen catheter and the VSSS were inserted separately into the mouth cavity, the nasal cavity, the tracheostomy tube, and the endotracheal tube of a human analog model for further comparisons. RESULTS: As the suction channel of the triple-lumen catheter was reduced by $46.8 \%$, the amount of simulant it suctioned was significantly less than that suctioned by the single-lumen catheter. However, under real-time guidance, the VSSS suctioned more simulant than the conventional single-lumen catheter in the human analog model. CONCLUSION: Sputum suctioning with the VSSS was feasible. Because of its real-time imaging guidance, the efficiency of the VSSS procedure was greater than that of the conventional single-lumen catheter. Therefore, this system may provide a new platform for sputum suctioning. Key words: suctioning; mисиs; sputum; feasibility; efficiency. [Respir Care 2013;58(10):1637-1642. (0) 2013 Daedalus Enterprises]
\end{abstract}

\section{Introduction}

Sputum suctioning is a routine clinical procedure that is crucial for keeping the airway open, to reduce the incidence of respiratory-tract infections. ${ }^{1}$ In most circumstances, a conventional catheter with a single-lumen channel is used for sputum suctioning. However, this procedure involves blind manipulation of the catheter on the part of the clinician and thus depends largely on the clinician's skills and experience. Therefore, complications occur in some patients, especially on the mucosa lining of the tra-

Drs Lv, Liu, Yan, and Deng are affiliated with the Department of Gerontology; Dr Wu is affiliated with the Department of Respiratory Medicine; and Dr Guo is affiliated with the Department of Critical Care Medicine, First Affiliated Hospital of Chongqing Medical University, Chongqing, China.

This research was partly supported by grant 2009AB5218 from the Chongqing Science and Technology Commission, and by grant 81201173 from the National Nature Science Foundation of China, neither of which had influence on the study design, data analysis, manuscript preparation, review, or authorization for submission. chea. In addition, because clinicians cannot directly target sputum in the trachea, the efficiency of the procedure may be reduced. ${ }^{2-6}$

Recently, a visual sputum suctioning system (VSSS) was developed in our research group. It integrates a $0.9-\mathrm{mm}$ micro-imaging fiber into a 5-mm triple-lumen catheter. The present study investigated the feasibility of sputum suctioning with this triple-lumen catheter alone in a laboratory setting. Furthermore, suctioning efficiency was evaluated in a human analog model to provide evidence of the applicability of this technique for clinical use. To the best

\footnotetext{
Supplementary material related to this paper is available at http:// www.rcjournal.com.
}

The authors have disclosed no conflicts of interest.

Correspondence: Huisheng Deng MD PhD, Department of Gerontology, First Affiliated Hospital of Chongqing Medical University, First You Yi Road, Chongqing 400016, China. E-mail: denghuisheng 2004@163.com.

DOI: $10.4187 /$ respcare. 01982 


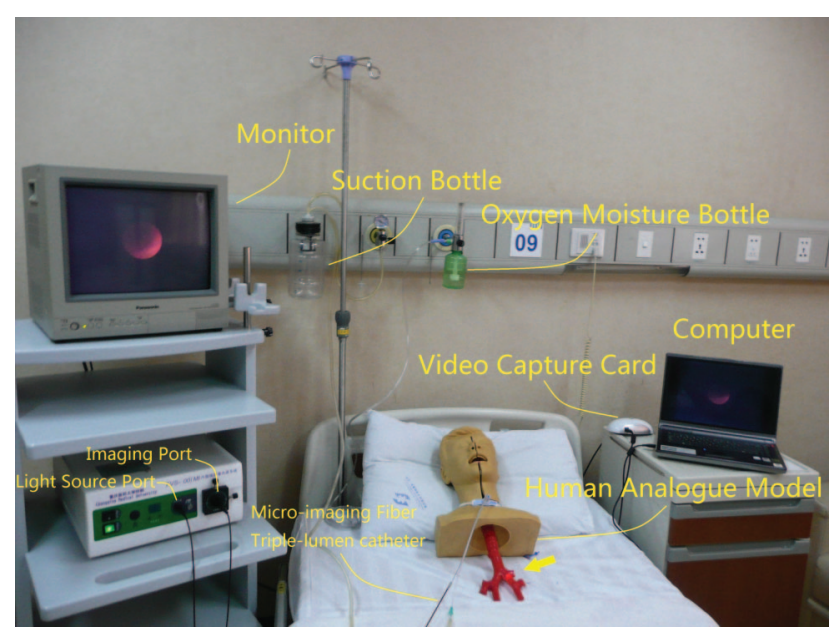

Fig. 1. Laboratory human analog model test setup for the visual sputum suctioning system. The arrow shows the red point as the distal end tip of the micro-imaging fiber in the trachea.

of our knowledge, this is the first study to evaluate sputum suctioning with the VSSS.

\section{Methods}

This study was conducted in the animal laboratory at First Affiliated Hospital of Chongqing Medical University, Chongqing, China.

\section{Visual Sputum Suctioning System}

The VSSS consisted of a micro-imaging fiber (FVS001MI, Blade, Beijing, China), a triple-lumen catheter (Tianjin Plastic Research Institute, Tianjin, China), and a computer processor and monitor (Fig. 1). The microimaging fiber has an image fiber bundle $(0.45 \mathrm{~mm})$ at its core, with a miniature optical lens at the distal end, and is surrounded by 120 optical fiber luminiferous bundles. The outer diameter of each optical fiber luminiferous bundle is $0.035 \mathrm{~mm}$. Including the coating layer and the optical fiber luminiferous bundle, the outside diameter of this microimaging fiber is $0.9 \mathrm{~mm}$, and the distal end can bend between about $0^{\circ}$ and $60^{\circ}$. The total length of the microimaging fiber is $220 \mathrm{~cm}$. To allow for deep suctioning, the length of the working portion is $50 \mathrm{~cm}$. The image from the lens has a resolution of 6,000 pixels. Depending on the guidance of the light source, the micro-imaging fiber records real-time images in the trachea, and the signals are processed and displayed on the computer monitor.

The triple-lumen catheter is $50 \mathrm{~cm}$ in length, with an outer diameter of $5 \mathrm{~mm}$ (Fig. 2). This results in an optical fiber channel with an inner diameter of $1.5 \mathrm{~mm}$ for the micro-imaging fiber, a suction channel with an inner diameter of $2.5 \mathrm{~mm}$, and an oxygen supply channel with an

\section{QUICK LOOK}

\section{Current knowledge}

Airway suctioning for secretion removal is typically a blind procedure, guided by patient response and successful suctioning of sputum. Suctioning is associated with various complications, some of which can be attributed to blind insertion and manipulation of the catheter.

\section{What this paper contributes to our knowledge}

A triple-lumen suction catheter that allows airway visualization was associated with improved secretion removal in a bench model system. Human studies and cost analysis are warranted.

inner diameter of $1.0 \mathrm{~mm}$. The conventional catheter has an outer diameter of $5 \mathrm{~mm}$ and a single-lumen channel (inner diameter $4.7 \mathrm{~mm}$ ) and is commercially available (Jiangsu Yongning Medical Apparatus Instrument, Jiangsu, China).

\section{Simulated Mucus/Sputum}

The simulated mucus/sputum was made with a water soluble, polyethylene oxide coagulant (Polyox NF, Dow Chemical, Midland, Michigan), which has an approximate molecular weight of 5,000,000 and a viscosity (1\% solution) of 5,500-7,500 centipoise. The simulated mucus/ sputum was prepared by heating water to $95^{\circ} \mathrm{C}$, quickly adding the coagulant, removing the mixture from the heat, and continuing to stir for 2 hours. We used $1.5 \%$ and $3.0 \%$ coagulant concentrations, which have properties comparable to human airway mucus and sputum. ${ }^{7}$

\section{Suctioning Tests With a Beaker}

We poured $150 \mathrm{~g}$ of the simulated mucus (coagulant concentration $1.5 \%$ ) or sputum (coagulant concentration $3.0 \%$ ) into a $250 \mathrm{~mL}$ beaker. The triple-lumen catheter was connected to the collection bottle and we suctioned the beaker for $15 \mathrm{~s}$ in each trial. ${ }^{8}$ The suctioning negative pressure was $200 \mathrm{~mm} \mathrm{Hg.9}$ The weight of simulant suctioned was calculated as the difference in the beaker's weight before and after suctioning. We followed the same procedure with the conventional single-lumen catheter. The average of 10 measurements was considered as the weight of the simulant suctioned by the catheters under the same conditions. 


\section{Laboratory Test of a Visual Sputum Suctioning System}

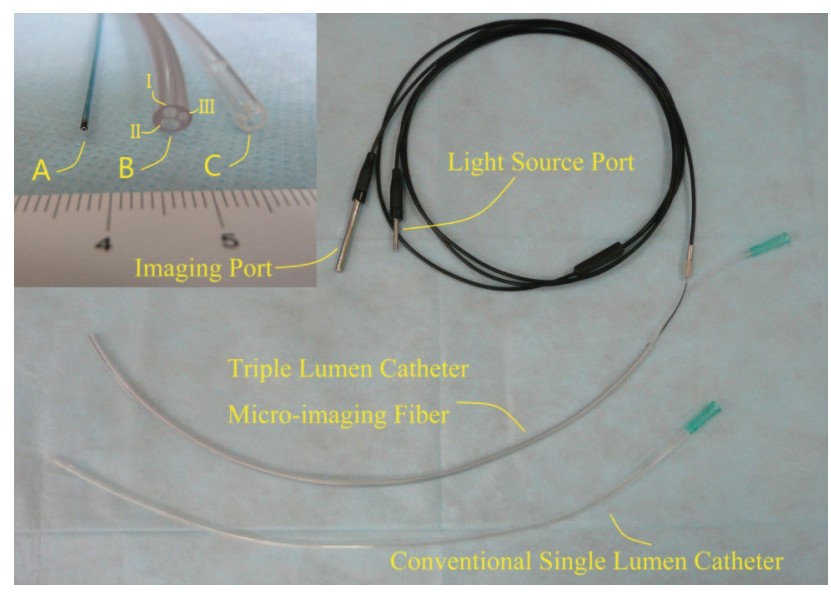

Fig. 2. The visual sputum suctioning system. A micro-imaging fiber is inserted into a triple-lumen catheter. The outer diameter of the triple-lumen catheter is equivalent to that of a conventional singlelumen catheter. A: Micro-imaging fiber (outer diameter $0.9 \mathrm{~mm}$ ). B: Triple-lumen catheter (outer diameter $5.0 \mathrm{~mm}$ ). I: Imaging channel (inner diameter $1.50 \mathrm{~mm}$ ). II: Suction channel (inner diameter $2.50 \mathrm{~mm}$ ). III: Oxygen supply channel (inner diameter $1.0 \mathrm{~mm}$ ). C: Conventional catheter (outer diameter $5.0 \mathrm{~mm}$ ) with a single lumen (inner diameter $4.7 \mathrm{~mm}$ ).

\section{Suctioning Tests With a Human Analog Model}

The first clinician randomly injected $20 \mathrm{~g}$ of the mucus simulant into the trachea of a human analog model (Zhonghong Teaching Equipment, Shanghai, China). The second clinician used the conventional single-lumen catheter to suction the simulant, with our routine suctioning procedure, for $15 \mathrm{~s}$ in each trial, ${ }^{8}$ with a suctioning negative pressure of $200 \mathrm{~mm} \mathrm{Hg} .{ }^{9}$ The third clinician used the VSSS with those same test conditions. The suctioning maneuver was performed in a standardized manner between the second and third clinicians. Preliminary tests found no significant difference in the weight of simulant suctioned by the conventional single-lumen catheter versus by the VSSS (data not shown).

In both test conditions the suctioning tube was inserted in separate tests into the trachea (via the mouth cavity), into the nasal cavity, into a tracheostomy tube (Smiths Medical, Kent, United Kingdom, inner diameter $7.5 \mathrm{~mm}$ ), and into an endotracheal tube (Mallinckrodt/Covidien, Mansfield, Massachusetts, inner diameter $7.5 \mathrm{~mm}$ ). The weight of simulant suctioned was calculated as the difference in the trachea's weight before and after suctioning.

For each suctioning route, 10 replications were performed, and we calculated the average amount of simulant suctioned. The simulant collected from both the conventional single-lumen catheter and the VSSS were randomly labeled, then all the simulant was measured by the first clinician, in a double-blind manner. Each procedure was evaluated by comparing the weights of the mucus simulant
Table 1. Weight of Simulated Mucus/Sputum Suctioned From the Beaker

\begin{tabular}{lcc}
\hline \hline & \multicolumn{2}{c}{ Weight, mean \pm SD g } \\
\cline { 2 - 3 } & $\begin{array}{c}\text { Simulated Mucus } \\
(1.5 \% \text { coagulant })\end{array}$ & $\begin{array}{c}\text { Simulated Sputum } \\
(3.0 \% \text { coagulant })\end{array}$ \\
\hline $\begin{array}{l}\text { Conventional single-lumen } \\
\text { catheter }\end{array}$ & $46.75 \pm 1.22$ & $53.97 \pm 2.18$ \\
$\begin{array}{l}\text { VSSS catheter } \\
* P<.001 .\end{array}$ & $35.42 \pm 1.56^{*}$ & $42.81 \pm 2.31 *$ \\
\begin{tabular}{l} 
VSSS = visual sputum suctioning system \\
\hline
\end{tabular}
\end{tabular}

collected in $15 \mathrm{~s}$ by the conventional catheter and the VSSS.

\section{Statistical Analysis}

We conducted the data analysis with statistics software (SPSS 10.0, SPSS, Chicago, Illinois). Data are presented as mean $\pm \mathrm{SD}$. We used the 2 sample $t$ test to compare the weights of simulant suctioned in the beaker experiments. We used factorial design analysis of variance to compare the weights of simulant suctioned in the human analog model. $P<.05$ was considered statistically significant.

\section{Results}

\section{Beaker Experiments}

We used a triple-lumen catheter to deliver the microimaging fiber into the suction catheter without interfering with the suction or compromising the oxygen supply. Although their outer diameters were the same, the inner diameter of the suction channel of the triple-lumen catheter was $46.8 \%$ less than that of the conventional single-lumen catheter. Because of the reduced size of the suction channel, the amounts of both the $1.5 \%$ and $3.0 \%$ simulant suctioned by the triple-lumen catheter were significantly lower than those suctioned by the conventional singlelumen catheter (Table 1) $(P<.001)$.

\section{Human Analog Model Experiments}

We used a human analog model to simulate the complicated anatomy of the human airway and to evaluate the use of the VSSS within the airway. As shown in Table 2, the VSSS was able to suction the $1.5 \%$ and $3 \%$ simulant from the mouth cavity, the nasal cavity, the tracheostomy tube, and the endotracheal tube. The VSSS was able to suction more simulant than the conventional single-lumen catheter (mouth cavity $P<.001$, nasal cavity $P<.001$, tracheostomy tube $P=.005$, endotracheal tube $P<.001$ ). 


\section{Laboratory Test of a Visual Sputum Suctioning System}

Table 2. Weight of Simulated Mucus/Sputum Suctioned From the Human Analog Model

\begin{tabular}{|c|c|c|c|c|}
\hline & \multicolumn{4}{|c|}{ Weight, mean \pm SD g } \\
\hline & \multicolumn{2}{|c|}{ Simulated Mucus ( $1.5 \%$ coagulant) } & \multicolumn{2}{|c|}{ Simulated Sputum ( $3.0 \%$ coagulant) } \\
\hline & $\begin{array}{l}\text { VSSS Catheter } \\
\qquad(n=10)\end{array}$ & $\begin{array}{c}\text { Conventional } \\
\text { Single-Lumen Catheter } \\
\quad(n=10)\end{array}$ & $\begin{array}{l}\text { VSSS Catheter } \\
\qquad(n=10)\end{array}$ & $\begin{array}{c}\text { Conventional } \\
\text { Single-Lumen Catheter } \\
(n=10)\end{array}$ \\
\hline Mouth cavity & $11.74 \pm 1.50^{*}$ & $9.80 \pm 1.00$ & $11.94 \pm 0.48^{*}$ & $9.03 \pm 1.07$ \\
\hline Nasal cavity & $10.46 \pm 1.03 *$ & $9.33 \pm 0.78$ & $9.96 \pm 1.00^{*}$ & $8.29 \pm 0.64$ \\
\hline Tracheostomy tube & $11.61 \pm 0.92^{*}$ & $10.41 \pm 0.94$ & $11.09 \pm 1.05^{*}$ & $10.55 \pm 0.68$ \\
\hline Endotracheal tube & $11.04 \pm 1.03 *$ & $9.34 \pm 0.72$ & $11.18 \pm 0.93^{*}$ & $8.21 \pm 1.07$ \\
\hline
\end{tabular}

\section{Discussion}

Our main results study are as follows:

- Both the triple-lumen and single-lumen catheters were able to suction simulant with properties equivalent to those of mucus and sputum.

- Because the suction channel of the triple-lumen catheter was $46.8 \%$ narrower than that of the single-lumen catheter, the amount of simulant suctioned by the triplelumen catheter was significantly less, under the same conditions.

- As the outer diameters of the single-lumen and triplelumen catheters were the same $(5 \mathrm{~mm})$, these catheters could be inserted into the airway of a human analog model from the mouth cavity, the nasal cavity, the tracheostomy tube, or the endotracheal tube.

- Under real-time imaging guidance, the VSSS collected more simulant than the conventional single-lumen catheter.

\section{Comparison of the VSSS and the Conventional Single-Lumen Catheter}

Sputum suctioning with a conventional single-lumen catheter is routine in clinical practice. However, as operators are not able to directly see what they are doing, this procedure depends largely on the experience of the clinician. This blind procedure can damage the trachea wall or decrease the effectiveness of the suctioning process. The visual guidance supplied by the VSSS enables the operator to estimate the amount and location of residual sputum after suctioning.

To address these limitations, we developed the VSSS (Figs. 1 and 2), which we evaluated for the first time in a laboratory setting in this study. Because of rapid progress in optical fiber technology, micro-imaging fibers as small as $0.9 \mathrm{~mm}$ are available. This micro-imaging fiber can be inserted into a conventional single-lumen catheter to guide suctioning. To further manage the oxygen supply and avoid disturbing the suctioning function, we used a triple-lumen catheter. Because both catheters had the same outer diameter $(5 \mathrm{~mm})$, the suction channel of the triple-lumen catheter was $46.8 \%$ less than that of the single-lumen catheter.

This study indicates that the inner diameter of the suction channel plays an important role in the efficiency of sputum suctioning (see Table 1). Under the same conditions, less sputum was suctioned by the triple-lumen catheter than the single-lumen catheter, because of the reduced inner diameter of the suction channel.

Although the beaker experiment suggests that the effectiveness of sputum suctioning with the triple-lumen catheter was significantly less than that of the conventional catheter (see Table 1), the human analog model experiment indicated that the VSSS was more efficient than the single-lumen catheter (see Table 2), because of the imaging guidance.

Sputum is randomly distributed within the airway, and to be as thorough as possible, conventional blind suctioning sometimes suctions trachea areas without sputum, which is inefficient and may cause unnecessary damage to the trachea. In contrast, imaging guidance can help the operator locate sputum quickly.

In this study the efficiency of sputum suctioning was determined by the weight of sputum suctioned; the suctioning time was not directly compared. However, it is possible that the greater efficiency of the VSSS may reduce suctioning time. Future studies should use animal models to evaluate the potential for reducing the risk of hypoxemia due to a long suctioning procedure. , $^{10}$

The VSSS catheter can be inserted into the airway more smoothly than a conventional suctioning catheter because the operator can visualize key anatomical structures such as the epiglottis, trachea wall, and carina (Fig. 3); this will reduce the incidence of airway damage. Future research 

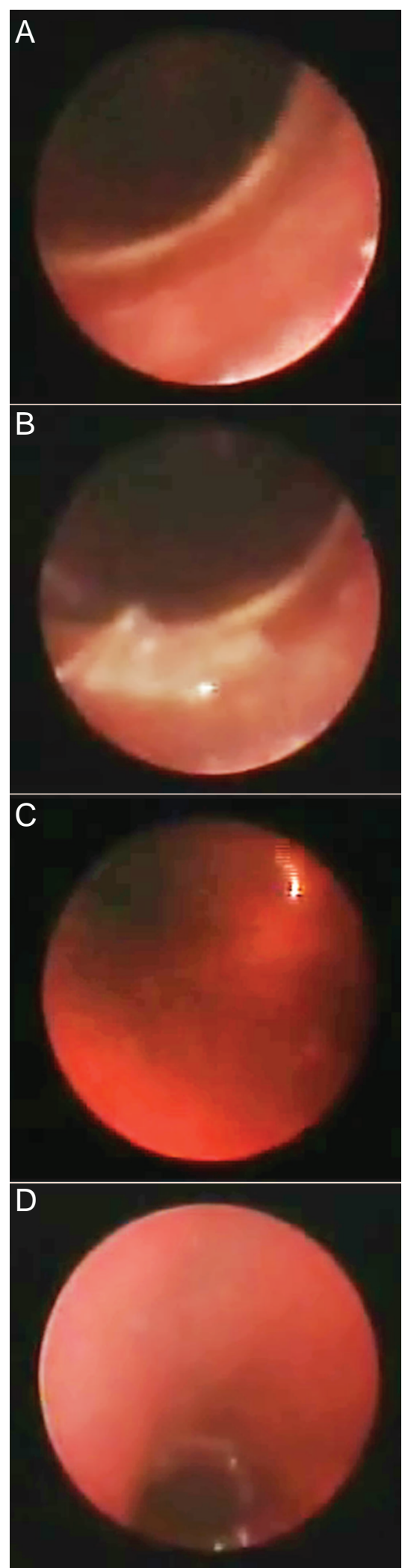

Fig. 3. Images from the visual sputum suctioning system in the human analog model. A: Trachea wall. B: Simulated mucus/ sputum in the trachea. C: Carina. D: Right bronchus. should use animal models or clinical trials to determine whether the VSSS has additional safety benefits.

\section{Comparison of the VSSS and the Bronchofiberscope}

Bronchofiberscopes are also used for suctioning in clinical practice. ${ }^{11,12}$ The commonly used 5-mm bronchofiberscope is equivalent to the triple-lumen catheter, so the VSSS represents an alternative to the bronchofiberscope.

Rapid progress in optical fiber technology reduced the diameter of the micro-imaging fiber to $0.9 \mathrm{~mm}$. Although optical imaging fiber bundles had previously been used in fiberoptic ductoscopy, to diagnose nipple discharge, ${ }^{13}$ the working length of the fiber was only $8.4 \mathrm{~cm}$. In order to enable deep suctioning within the trachea, we extended the optical fiber to $50 \mathrm{~cm}$. To the best of our knowledge, this is the first report to demonstrate the application of imaging guidance for sputum suctioning using an optical fiber of such length.

The image resolution of the micro-imaging fiber was 6,000 pixels, which was about one fifth less than that of the bronchofiberscope. ${ }^{14}$ However, the primary purpose of this study was to use the micro-imaging fiber to guide suctioning, not for diagnosis, and the quality of the images acquired by the VSSS was sufficient for this purpose (see the supplementary materials at http://www.rcjournal.com).

In China, only clinicians and respiratory therapists are allowed to use the bronchofiberscope; moreover, repeated sterilization is necessary within different patients, which limits the application of this technique for routine clinical practice and repeated suctioning. In contrast, sterilization involves simply changing the triple-lumen catheter. Also, as the basic procedure for sputum suctioning using the VSSS is similar to the conventional method, respiratory therapists are able to perform this procedure competently, which may help to further popularize this new method.

\section{Implications for Clinical Practice}

In this study a micro-imaging fiber was inserted into a triple-lumen catheter to guide sputum suctioning. In addition to its use in suctioning, this micro-imaging fiber has the potential to be integrated into the nasogastric tube, urethral catheter, or rubber drainage tube. Therefore, this technique has the potential to change and improve many clinical practices.

\section{Limitations}

Although the VSSS has many potential benefits, this prototype system is still in its infancy. Further optimization in terms of catheter design and manipulation and resolution of the micro-imaging fiber should take place according to the results of subsequent studies. Moreover, 


\section{Laboratory Test of a Visual Sputum Suctioning System}

because of the external diameter of the catheter, this system is not suitable for suctioning sputum in smaller endotracheal tubes (eg, those of neonates and pediatric patients).

Future studies should investigate the efficiency and efficacy of the VSSS in animal models to further evaluate the safety of this technique. An application for a clinical trial is also pending. Studies of this system in patients will provide the most meaningful information on the feasibility, efficiency, and safety of this technique; the perspectives of patients; the acceptance of the technique among clinicians; and the financial benefits of this system.

\section{Conclusions}

The VSSS is feasible for sputum suctioning in a laboratory setting. Although its efficiency was reduced, compared to that of a triple-lumen catheter in vitro, this drawback can be compensated for by the targeted sputum suctioning allowed by imaging guidance. Therefore, the VSSS may provide a new platform for sputum suctioning.

\section{REFERENCES}

1. Choi JS, Jones AY. Effects of manual hyperinflation and suctioning in respiratory mechanics in mechanically ventilated patients with ventilator-associated pneumonia. Aust J Physiother 2005;51(1):25-30.

2. Yorioka K, Oie S, Kamiya A. Microbial contamination of suction tubes attached to suction instruments and preventive methods. Jpn J Infect Dis 2010;63(2):124-127.

3. Barnes CA, Kirchhoff KT. Minimizing hypoxemia due to endotracheal suctioning: a review of the literature. Heart Lung 1986;15(2): 164-176.
4. Brochard L, Mion G, Isabey D, Bertrand C, Messadi AA, Mancebo J, et al. Constant-flow insufflation prevents arterial oxygen desaturation during endotracheal suctioning. Am Rev Respir Dis 1991; 144(2):395-400.

5. Maggiore SM, Lellouche F, Pigeot J, Taillé S, Deye N, Durrmeyer $\mathrm{X}$, et al. Prevention of endotracheal suctioning-induced alveolar derecruitment in acute lung injury. Am J Respir Crit Care Med 2003; 167(9):1215-1224

6. Almgren B, Wickerts CJ, Heinonen E, Hogman M. Side effects of endotracheal suction in pressure- and volume-controlled ventilation. Chest 2004;125(3):1077-1080.

7. Shah S, Fung K, Brim S, Rubin BK. An in vitro evaluation of the effectiveness of endotracheal suction catheters. Chest 2005;128(5): 3699-3704.

8. Pedersen CM, Rosendahl-Nielsen M, Hjermind J, Egerod I. Endotracheal suctioning of the adult intubated patient: what is the evidence? Intensive Crit Care Nurs 2009;25(1):21-30.

9. Chao ZZ. The progress of artificial airway suction nursing. Nurs Pract Res 2008;5(4):73-74.

10. El Masry A, Williams PF, Chipman DW, Kratohvil JP, Kacmarek RM. The impact of closed endotracheal suctioning systems on mechanical ventilator performance. Respir Care 2005;50(3):345-353.

11. Kenzaki K, Hirose Y, Tamaki M, Sakiyama S, Kondo K, Mutsuda T, et al. Novel bronchofiberscopic catheter spray device allows effective anesthetic spray and sputum suctioning. Respir Med 2004;98(7): 606-610.

12. Van de Leur JP, Zwaveling JH, Loef BG, Van der Schans CP. Endotracheal suctioning versus minimally invasive airway suctioning in intubated patients: a prospective randomised controlled trial. Intensive Care Med 2003;29(3):426-432.

13. Liu GY, Lu JS, Shen KW, Wu J, Chen CM, Hu Z, et al. Fiberoptic ductoscopy combined with cytology testing in the patients of spontaneous nipple discharge. Breast Cancer Res Treat 2008;108(2):271-277.

14. Kaneko M. Changes and current state of diagnosis of lung cancer after development of the flexible bronchofiberscope. Jpn J Clin Oncol 2010;40(9):838-845. 